\title{
ANÁLISE QUÍMICA DO BAMBU-GIGANTE (Dendrocalamus giganteus Wall. ex Munro) EM DIFERENTES IDADES
}

\author{
CHEMICAL ANALYSIS OF GIANT BAMBOO \\ (Dendrocalamus giganteus Wall. ex Munro) AT DIFFERENT AGES
}

\author{
Nelson Potenciano Marinho ${ }^{1}$ Silvana Nisgoski ${ }^{2}$ Umberto Klock ${ }^{3}$ \\ Alan Sulato de Andrade ${ }^{4}$ Graciela Inês Bolzon de Muñiz ${ }^{5}$
}

\begin{abstract}
RESUMO
O bambu é uma planta monocotiledônea de origem tropical, de rápido crescimento, sendo considerado um importante regenerador ambiental devido ao seu sistema radicular. O Dendrocalamus giganteus é popularmente conhecido por bambu-gigante, sendo uma espécie entouceirante de grande porte, cujos colmos podem variar de 24 a 40 metros de altura, com diâmetros entre 10 e $20 \mathrm{~cm}$. Esse trabalho teve como objetivo analisar quimicamente os colmos do bambu-gigante visando fornecer informações sobre a sua composição química nas idades de 2 a 6 anos. Os colmos do bambu foram coletados na área Experimental Agrícola do Departamento de Engenharia Mecânica da UNESP-Bauru, onde é realizado o manejo sistemático de diversas espécies de bambu para fins de pesquisas e divulgação dessa planta. Foram retiradas amostras da base entre bambus com idade de 2 a 6 anos de uma mesma touceira, totalizando 5 amostras, com 2 metros de altura cada, colhidas na lua minguante no mês de março. As análises químicas foram feitas com os entrenós, utilizando as normas e procedimentos da TAPPI. A porcentagem média de extrativos em água quente, extrativos em água fria, extrativos totais e teor de cinzas não apresentaram relação direta com a idade. A maior porcentagem de extrativos em água quente, com valor de $12,04 \%$, extrativos em água fria, com 10,25\%, e extrativos totais, com 12,91\%, foi observada aos 2 anos de idade. A maior porcentagem de cinzas foi obtida aos três anos, com valores de 1,09\%. O teor médio de extrativos em $\mathrm{NaOH}$ diminui de $25,72 \%$ aos dois anos para $20,17 \%$ aos seis anos de idade. O mesmo comportamento foi observado para os extrativos em etanol tolueno, diminuindo de $10,91 \%$ para 4,61\%. O teor médio de lignina não sofreu influência da idade, variando de 22,66 a 24,11\%.
\end{abstract}

Palavras-chave: Dendrocalamus giganteus; extrativos; lignina; cinzas.

\begin{abstract}
Bamboo is a monocotyledon of tropical origin which grows fast and is considered an important environment regenerator because of its root system. Dendrocalamus giganteus is popular learned by giant bamboo, being a big bush species, its culms can be from 24 to 40 meters high, with diameters from 10 and 20 centimeters. This work had the objective of a chemical analysis from giant bamboo to aim at giving information from its chemical composition from 2 to 6 years of age. Bamboo culms were collected in the Agriculture Experimental area of the Mechanical Engineering Department of UNESP - Bauru, where it is carried out the systematic handling from different bamboo species to this plant research and divulgation. Samples from
\end{abstract}

1. Designer, Mestrando do Programa de Pós-graduação em Engenharia de Materiais, Universidade Tecnológica Federal do Paraná, Av. Sete de Setembro, 3165, Rebouças, CEP 80230-901, Curitiba (PR). n.pmarinho@yahoo.com.br

2. Engenheira Florestal, Dra ${ }^{a}$, Professora do Departamento de Engenharia e Tecnologia Florestal, Universidade Federal do Paraná, Av. Pref. Lothário Meissner, 900, CEP 80210-170, Curitiba (PR). nisgoski@upfr.br

3. Engenheiro Florestal, Dr., Professor do Departamento de Engenharia e Tecnologia Florestal, Universidade Federal do Paraná, Av. Pref. Lothário Meissner, 900, CEP 80210-170, Curitiba (PR). klockuer@upfr.br

4. Engenheiro Florestal, Dr., Professor do Departamento de Engenharia e Tecnologia Florestal, Universidade Federal do Paraná, Av. Pref. Lothário Meissner, 900, CEP 80210-170, Curitiba (PR). alansulato@upfr.br

5. Engenheira Florestal, Dra ${ }^{a}$, Professora do Departamento de Engenharia e Tecnologia Florestal, Universidade Federal do Paraná, Av. Pref. Lothário Meissner, 900, CEP 80210-170, Curitiba (PR). gbmunize@upfr.br Recebido para publicação em 11/06/2010 e aceito em 04/05/2011 
base were collected from 2 and 6 years in the same bush, in total of 5 samples, with 2 high meters each, cropped in March, half moon. Chemical analysis was done with internodes using TAPPI Methods. The average percentage extractives from hot water, cool water, total extractives and ash content were not related to the age. The major percentage of hot water extractives, with values of $12.04 \%$, cold water extractives, with $10.25 \%$, and total extractives, with $12.91 \%$, was observed by the age of two years. The major ash content was obtained on three years, with value of $1.09 \%$. The mean value of $\mathrm{NaOH}$ extractives decreased from $25.72 \%$, in two years, to $20.17 \%$ at six years old. The same behavior was observed for ethanol toluene extractives, decreasing from $10.91 \%$ to $4.61 \%$. The mean lignin content was not influenced by age, varying from 22.66 to $24.11 \%$.

Keywords: Dendrocalamus giganteus; extractives; lignin; ash.

\section{INTRODUÇÃO}

O bambu pertence à família das Poaceae, subfamília Bambusoideae com aproximadamente 75 gêneros e mais de 1.250 espécies(LONDOÑO, 2004). São plantas anuais ou perenes, às vezes lignificadas, em geral rizomatosas e providas de colmos ocos ou sólidos (SILVA FILHO, 2006). Segundo o sistema de classificação do botânico alemão Adolf Engler, o bambu pertence à divisão das Angiospermae e faz parte do grupo das monocotiledôneas, subgrupo das Gramineae, sendo parente próximo do trigo, milho, cana-de-açúcar, cevada e capim (OSTAPIV, 2007). As espécies exóticas, introduzidas, por intermédio dos imigrantes, são, na sua maioria, provenientes do continente asiático, tais como: Bambusa vulgaris (sul da China), Bambusa tuldoides (sul Asiático), Dendrocalamus strictus (Índia, China e Vietnã), Dendrocalamus giganteus (Burma) e Phyllostachys aurea (Ásia) (AZZINI et al., 1997).

Trata-se de uma planta de origem tropical, perene, renovável, e capaz de produzir novos brotos sem a necessidade do replantio. Outra característica que diferencia o bambu de outras plantas é o seu rápido crescimento. Azzini et al. (1989), estudando o crescimento do bambu nas condições de clima e solo do Centro Experimental de Campinas (IAC), verificaran que a velocidade máxima de crescimento axial nos colmos das espécies Guadua angustifolia e Dendrocalamus giganteus variou de 7,88 a $22,00 \mathrm{~cm} /$ dia. Jaramillo (1992) afirma que não há nenhuma outra espécie florestal que possa competir em velocidade de crescimento e de aproveitamento, sendo o bambu um importante regenerador ambiental devido ao seu sistema radicular, podendo ser utilizado em reflorestamento.

O bambu-gigante (Dendrocalamus giganteus) é uma espécie entouceirante de grande porte, onde na mesma "touceira" convivem diversos colmos de diferentes idades, que podem variar de 24 a 40 metros de altura, com diâmetros entre 10 e 20 $\mathrm{cm}$ e espessura de parede entre 1 a $3 \mathrm{~cm}$ (PEREIRA e BERALDO, 2007). Originário da Malásia, possui folhas acuminadas e flores verdes de início, que depois se tornam amareladas e pardo-claras, com espiguetas paniculadas, a planta floresce a cada trinta anos.

Apesar do grande potencial do bambu, ele é ainda pouco utilizado industrialmente, o que de acordo com Pereira e Beraldo (2007), é decorrente do desconhecimento da variedade de suas espécies, das suas propriedades características associadas a diversos usos e aplicações, devido à falta de pesquisas específicas e a insuficiente informação ao seu respeito. De acordo Londoño et al. (2002), a química e os padrões estruturais nos colmos são propriedades bastante significativas, quando correlacionados com a idade. Liese e Weiner (1996) afirmam que a porcentagem de holocelulose, em bambus com mais de 1 ano, tende a diminuir, enquanto a lignina se mantém estável, tendendo a aumentar um pouco.

Portanto, o conhecimento da composição química dos colmos de bambu das diversas espécies é importante para obtenção de dados relativos às características peculiares de cada uma, sendo uma fonte de informação utilizada pela botânica para ajudar na identificação e classificação das espécies, além de elucidar as correlações entre as suas diversas propriedades.

De acordo com Liese (1998) e Lopez (2003), as propriedades físicas e mecânicas dos bambus estão correlacionadas com as suas propriedades químicas e anatômicas, destacando-se o teor de lignina e a espessura das paredes celulares. Kollmann e Coté (1968) afirmam que as oscilações na densidade dos tecidos lenhosos também são originárias das diferenças estruturais, anatômicas e pela presença de extrativos na sua composição química. A composição química do bambu, de acordo com Higuchi (1990), é semelhante à da madeira, com exceção dos extratos 
alcalinos, cinzas e sílica, que são maiores no bambu. Assim, os principais constituintes químicos dos colmos de bambu são a celulose, holocelulose e lignina e, em menor escala, estão presentes as resinas, tanino (ácido tânico), ceras e sais orgânicos (COSTA, 2004), sendo que a celulose com $55 \%$ e a lignina com $25 \%$ são os constituintes em maior quantidade (PEREIRA e BERALDO, 2007).

A lignina é um constituinte da parede celular e ocorre na maioria das plantas, funcionando como elemento de suporte, ou seja, é um elemento estrutural que proporciona à planta propriedades de elasticidade e resistência, mas sua composição não é idêntica em todas as plantas. É um polímero de alto peso molecular, formado por unidades fenilpropanóides, que, após a polimerização na parede celular, tornam-se menos complexas e resistentes (ABREU et al., 1999). Liese (1985a) afirma que a lignina é constituída nos bambus a partir do 3-fenil-p-propano e unidades p-cumaril, coniferil e álcoois sinapílicos interligados por vias biossintéticas.

Os diversos estágios de lignificação estão relacionados às fases de crescimento da planta, que começam da base seguindo em direção ao topo no colmo. Pereira e Beraldo (2007) afirmam que a lignificação dos entrenós é diferente, ou seja, no sentido longitudinal, ela ocorre do topo em direção à base, enquanto que no sentido transversal ocorre de dentro para fora, completando todo o processo dentro do estágio de crescimento. O mesmo autor afirma que a proporção de lignina e de carboidratos varia durante o período de maturação, e que tende a estabilizar-se em torno de um ano. Os teores de substâncias solúveis (resinas, ácidos graxos, óleos essenciais, tanino, etc.), de cinzas e de lignina são menores nos nós, contudo, os teores de celulose são maiores em relação aos entrenós.

Os fatores climáticos também influenciam na composição química do bambu, principalmente na quantidade de extrativos totais. Devido à facilidade de serem extraídos em água ou em solventes orgânicos neutros, ou volatilizados através do vapor, os extrativos vegetais são compostos orgânicos aromáticos (ácidos graxos, terpenos, flavonoides, esteroides, etc.) que não fazem parte da parede celular lignocelulósica. Os extrativos estão em toda a parte da planta (casca, folhas, frutos, caule e sementes) e sua ocorrência varia de acordo com a espécie vegetal, sendo que nas fibras variam de 3 a 10\% (D'ALMEIDA et al., 1988).

A quantidade de substâncias inorgânicas como: potássio, cálcio, silício e magnésio, é denominada como teor de cinzas. Os nós do bambu apresentam maior quantidade de celulose e menos extrativos solúveis em água, hemicelulose (pentoses), cinzas e lignina (BAI, 1996). Estudos realizados por Chen et al. (1985) indicam que a composição das cinzas varia, significativamente, entre os colmos de 1 a 7 anos de idade, sendo observada uma diminuição nos teores de cobre, zinco, fósforo, ferro e potássio, e um aumento nos teores cálcio, magnésio e manganês. Conforme Liese (1985b) as substâncias inorgânicas (1 a 5\%) estão presentes em maior quantidade nos internós nas camadas mais internas do colmo e o teor de silício $(0,5$ a $4 \%)$ está concentrado na epiderme dos internós e bem pouco nos nós. Liese (1992) afirma que o silício afeta na qualidade da polpação, variando entre 0,5 a $5 \%$, de acordo com a espécie.

Portanto, esse trabalho teve como objetivo analisar quimicamente os colmos do bambu Dendrocalamus giganteus, visando fornecer informações sobre a sua composição química nas idades de 2 a 6 anos, em função da existência de poucos estudos com esse enfoque.

\section{MATERIAL E MÉTODOS}

Os colmos do bambu (Dendrocalamus giganteus) utilizados nesse trabalho foram coletados na área Experimental Agrícola do Departamento de Engenharia Mecânica da UNESP-Bauru, em São Paulo, local onde é realizado o manejo sistemático de diversas espécies de bambu para fins de pesquisas científicas. Foram retiradas amostras da base de bambus com idade entre 2 a 6 anos de uma mesma touceira, totalizando 5 amostras, com 2 metros de altura cada.

As análises químicas foram feitas com os entrenós utilizando as normas e procedimentos da TAPPI (1994) para Extrativos em Água Quente (T264 om-88), Extrativos em Água Fria (T207 om93), Extrativos em $\mathrm{NaOH}$ (T 212 om-93), Extrativos em Etanol Tolueno (T204 om-88), Extrativos Totais (T204 om94), Teor de Cinzas (T211-om02), Teor de Lignina (T222-om02).

\section{RESULTADOS E DISCUSSÕES}

A média dos resultados obtidos nas análises químicas dos entrenós do Dendrocalamus giganteus, retirados próximo da base (até 2 metros de altura) com idades entre 2 a 6 anos, são apresentados na Tabela 1. 
TABELA 1: Análises químicas do bambu (Dendrocalamus giganteus), com idades entre 2 e 6 anos, da região de Bauru (São Paulo - Brasil).

TABLE 1: Bamboo chemical analysis (Dendrocalamus giganteus), with age between 2 and 6 years, from Bauru region (São Paulo - Brazil).

\begin{tabular}{cccccccc}
\hline \multirow{2}{*}{$\begin{array}{c}\text { Idade } \\
\text { (anos) }\end{array}$} & $\begin{array}{c}\text { Extrativos } \\
\text { em água } \\
\text { quente }(\%)\end{array}$ & $\begin{array}{c}\text { Extrativos } \\
\text { em água fria } \\
(\%)\end{array}$ & $\begin{array}{c}\text { Extrativos } \\
\text { em NaOH } \\
(\%)\end{array}$ & $\begin{array}{c}\text { Extrativos } \\
\text { em Etanol } \\
\text { Tolueno }(\%)\end{array}$ & $\begin{array}{c}\text { Extrativos } \\
\text { Totais }(\%)\end{array}$ & $\begin{array}{c}\text { Teor de } \\
\text { Cinzas } \\
(\%)\end{array}$ & $\begin{array}{c}\text { Teor de } \\
\text { Lignina } \\
(\%)\end{array}$ \\
\hline 2 & $12,04 \mathrm{a}$ & $10,25 \mathrm{a}$ & $25,72 \mathrm{a}$ & $10,91 \mathrm{a}$ & $12,91 \mathrm{a}$ & $0,74 \mathrm{a}$ & $22,66 \mathrm{a}$ \\
3 & $9,62 \mathrm{ab}$ & $7,96 \mathrm{~b}$ & $24,04 \mathrm{bc}$ & $7,30 \mathrm{~b}$ & $9,21 \mathrm{bc}$ & $1,09 \mathrm{~b}$ & $22,77 \mathrm{a}$ \\
4 & $7,06 \mathrm{bc}$ & $4,61 \mathrm{c}$ & $21,01 \mathrm{bc}$ & $7,93 \mathrm{bc}$ & $10,27 \mathrm{~b}$ & $0,66 \mathrm{ac}$ & $23,48 \mathrm{a}$ \\
5 & $10,02 \mathrm{ab}$ & $8,14 \mathrm{~b}$ & $21,64 \mathrm{bcd}$ & $6,32 \mathrm{bcd}$ & $9,93 \mathrm{~b}$ & $0,64 \mathrm{ac}$ & $24,11 \mathrm{a}$ \\
6 & $7,25 \mathrm{c}$ & $4,86 \mathrm{c}$ & $20,17 \mathrm{e}$ & $4,61 \mathrm{~d}$ & $7,87 \mathrm{c}$ & $0,84 \mathrm{a}$ & $23,28 \mathrm{a}$ \\
\hline
\end{tabular}

Em que: Médias seguidas pela mesma letra na coluna não diferem entre si pelo teste de Tukey a $95 \%$ de probabilidade.

Os maiores teores de extrativos em água quente, água fria, $\mathrm{NaOH}$, etanol tolueno e extrativos totais foram obtidos nas análises das amostras com dois anos de idade, enquanto que os menores teores de extrativos em água quente, água fria e $\mathrm{NaOH}$ foram obtidos aos quatro anos, e de extrativos em etanol tolueno e totais, aos seis anos. No caso do teor de cinzas, a idade de três anos apresentou o maior percentual médio e em relação ao teor de lignina o maior valor foi encontrado para os bambus com cinco anos.

Também se pode observar que os resultados obtidos para a porcentagem de extrativos em água quente, água fria e totais, além do teor de cinza, não apresentaram relação direta com a idade. Ao contrário, a porcentagem de extrativos em $\mathrm{NaOH}$ e em etanol tolueno diminui com o aumento da idade. As variações são explicadas pelas diferenças na composição química das substâncias extraídas, como cera, resina, tanino e sais orgânicos (LIESE, 1998).

O percentual de cinzas obtido nesse estudo ficou abaixo da média da espécie encontrada na literatura (PROTA, 2010; LWIN et al, 2010), podendo ser resultado da variação da altura de amostragem, espessura do colmo e posição da amostra na touceira. Outros fatores também podem estar relacionados como o manejo, o clima e a quantidade de nutrientes disponíveis para o desenvolvimento de cada amostra.

$O$ teor de lignina não sofreu influência da idade, o que comprova as informações encontradas na literatura (PEREIRA E BERALDO, 2007), que relatam que a porcentagem deste componente estabiliza depois da maturidade, em torno de um ano de idade, dependendo da espécie.
Segundo informações publicadas no guia Plant Resources of Tropical Africa (PROTA, 2010), o bambu Dendrocalamus giganteus da região de Madagascar (África), sem identificação de idade, após análises químicas apresentou os seguintes resultados: para os extrativos em água quente 5,1\%, teor de cinzas $2,9 \%$, teor de lignina $25,3 \%$, extrativos etanol benzeno $6,5 \%$ e extrativos em $\mathrm{NaOH} 24,4 \%$. Os valores de extrativos em água quente dos estudos de Madagascar são bem inferiores, e o teor de cinzas é superior aos resultados obtidos neste estudo, independente da idade, enquanto os outros resultados mostram-se próximos.

Lopez (1974), estudando diversas espécies de bambu para a aplicação em projetos arquitetônicos, analisou quimicamente, dentre outras, o Dendrocalamus strictus proveniente da Índia, obtendo os seguintes resultados: teor de extrativos em $\mathrm{NaOH}$ de $16,4 \%$, em água quente $9,7 \%$, teor de lignina de $14,4 \%$ e cinzas $2,5 \%$ para o bambu maduro (até 1 ano) e teor de extrativos em $\mathrm{NaOH}$ de $14,4 \%$, em água quente $3,0 \%$, teor de lignina de $17,8 \%$ e cinzas $1,0 \%$, para o bambu com 4 anos. Lwin et al. (2010), caracterizando quimicamente diferentes bambus de Myanmar, encontrou valores de: teor de cinzas de 1,9\%, extrativos em água quente de $16,6 \%$, em $\mathrm{NaOH}$ de $28,4 \%$, em álcool benzeno de $7,4 \%$ e teor de lignina de $17,8 \%$, para Dendrocalamus giganteus com 3 anos de idade. Os resultados descritos acima para essa espécie seguem a mesma tendência dos obtidos para o Dendrocalamus giganteus neste estudo.

Gomide et al. (1988), ao analisar quimicamente o bambu da espécie Bambusa vulgaris com a finalidade de avaliar suas características para a produção de papel na região de São Paulo, 
obteve os seguintes resultados: $15 \%$ para extrativos em água quente, $33,4 \%$ para extrativos em $\mathrm{NaOH}$, $5,2 \%$ para etanol benzeno, $1,8 \%$ para teor de cinzas, e $14,5 \%$ para teor de lignina.

A composição química do Dendrocalamus giganteus obtida neste estudo, avaliando diferentes idades, apresenta variações no teor de lignina, extrativos e cinzas em relação a outros estudos, o que pode ser justificado em função das condições de crescimento das touceiras, a idade dos colmos, região do colmo analisada e a estação do ano em que foi efetuada a coleta, como comentado por Beraldo e Azzini (2004).

\section{CONCLUSÕES}

Não foi observada variação estatística significativa no teor de lignina entre as idades analisadas, ou seja, entre 2 e 6 anos.

Os teores de extrativos em água quente, água fria e extrativos totais, além do teor de cinza, apresentaram elevada variação entre as idades, mas não seguiram nenhum padrão.

A porcentagem de extrativos em $\mathrm{NaOH}$ e em etanol tolueno diminuiu à medida que a idade aumentou, indicando a influência da idade na quantidade de extrativos.

Como o Dendrocalamus giganteus é uma espécie de uso comercial, sendo muito utilizado na fabricação de painéis, há necessidade de maiores investigações a respeito das interações existentes entre os diversos tipos de adesivo e a composição química da espécie estudada, em relação ao tipo e quantidade de extrativos.

\section{REFERÊNCIAS BIBLIOGRÁFICAS}

ABREU, H. S.; NASCIMENTO, A. M.; MARIA, M. A. Lignin and structure. Wood and Fiber Science, v. 31, n. 4, p. 426-433, Oct. 1999.

AZZINI, A.; CIARAMELO, D.; SALGADO, A.L.B. Velocidade de crescimento dos colmos de algumas espécies de bambu. O Agronômico, Campinas, v. 41, n. 3, p. 199-200, set-dez, 1989.

AZZINI, A.; SANTOS, R.L.; PETTINELLI JÚNIOR, A. Bambu: material alternativo para construções rurais. Campinas, SP, Instituto Agronômico de Campinas, Boletim Técnico, n. 171, p. 1-18, 1997.

BAI, X. Experimental and numerical evaluations of structural bamboo-based composite materials. 1996. 163 p. Dissertation (MS in Forest Resources)-
Clemson University Clemson, 1996.

BERALDO, A. L.; AZZINI, A. Bambu: características e aplicações. Guaíba: Agropecuária, 2004.

CHEN, Y. et al. The chemical composition of ten bamboo species. In Rao, A.N; Dhanarajan, G.; Sastry, C.B. ed., Recent Research on Bamboo. Proceedings of the International Bamboo Workshop, Hangzhou, China, 6-14 October 1985. Chinese Academy of Forestry, Beijing, China; International Development Research Centre, Ottawa, Canada. p. 110-113. COSTA, T.M. de S. Estudo da viabilidade técnica do emprego do bambu da espécie Bambusa vulgaris Schard. como carvão vegetal. São Paulo: USP, 62p. Dissertação (Mestrado em Ciências na área de Tecnologia Nuclear - Materiais). Instituto de Pesquisa Energéticas e Nucleares-IPEN, São Paulo, 2004.

D'ALMEIDA, M.L.O et al. Celulose e papel: tecnologia da fabricação de pasta celulósica. Vol. 1, 2.ed. São Paulo: Senai/IPT, 1988. 559 p.

GOMIDE, J.L.; VIVONE, R.R.; GALA, P.A.M. Bambu: uma alternativa para o déficit de celulose de fibra longa no Brasil. In: CONGRESSO ANUAL DE CELULOSE E PAPEL DA ABCP, 21. São Paulo, 1988. Anais... Associação Brasileira de Celulose e Papel: São Paulo, 1988, p.1-16.

HIGUCHI, T. Chemistry and biochemistry of bamboo. Wood Research Institute, Kyoto University, Kyoto, Japan, 1990.

JARAMILLO, S. V. La Guadua em los projectos de inversión. In: CONGRESSO MUNDIAL DE BAMBU/GUADUA, 1992, Colômbia. Anais... Pereira, 1992.

KOLLMANN, F.F.P.; CÔTÉ Jr, W.A. Principles of wood science and technology. New York: Springer Verlag, 1968. v. 1: Solid Wood. 592 p.

LIESE, W. The structure of bamboo in relation to its properties and utilization. In: International Symposium on Industrial use of bamboo. Intern. Trop. Timber Organization, Chinese Academy of Forestry. Beijing, China. 1992.

LIESE, W. Anatomy and properties of bamboo, recent research on bamboo. Proceedings of the INTERNATIONAL BAMBOO WORKSHOP, $1985 \mathrm{a}$.

LIESE, W. Bamboos: Biology, silvics, properties, utilization, Eschborn, Deutsche Gesellschaft fur Technische Zusammenarbeit (GTZ), 1985b, p. 5152 e $72-74$.

LIESE, W. The anatomy of bamboo culms. Technical Report. 1998. 207p. 
LIESE, W.; WEINER, G. Ageing of Bamboo culms. Proceeding of the V INTERNATIONAL BAMBOO CONGRESS. 2-6 November 1996. Costa Rica.

LONDOÑO, X. Distribuição, morfologia, taxonomia, anatomia, silvicultura y usos de los bambues de lo Nuevo Mundo. In: III CONGRESO COLOMBIANO DE BOTÂNICA, 2004, Popayan. Anais... Popayan, 2004. 25p.

LONDOÑO, X. et al. Characterization of the anatomy of Guadua angustifolia (Poaceae: Bambusoideae) culms. Bamboo Science and Culture: The Journal of the American Bamboo Society, v.16, n.1, p.18-31, 2002.

LOPEZ, O. H. Bamboo, The gift of the gods. Bogotá: Ed. D’Vinni, 2003. 553p.

LOPEZ, O. H. Bambú su cultivo y aplicaciones en: fabricación de papel, construcción, arquitectura, ingeniería, artesanía. Colômbia: Estúdios Técnicos Colombianos Ltda., 1974.318p.

LWIN, K.M. et al. An investigation on morphology, anatomy and chemical properties of some Myanmar bamboos. Disponível: www.myanmar. gov.mm/AgJurnal/ProcFo01-10pdf. Acesso em 22 de fevereiro de 2010.

OSTAPIV, F. Analise e melhoria do processo produtivo de tábuas de bambu (Phyllostachys pubences) com foco em pisos. Curitiba: UTFPR, 2007. 116p. Dissertação (Mestrado em Engenharia Mecânica e de Materiais). Universidade Tecnológica Federal do Paraná, 2007.

PEREIRA, M. A. R.; BERALDO, A.L. Bambu de corpo de alma. Bauru, SP: Editora Canal 6, 2007. 239p.

PROTA. Plant Resources of Tropical Africa. PROTA 7 (1): Madeira. Disponível: http://database.prota. org/PROTAhtml/Dendrocalamus\%20giganteus

En.htm. Acesso em 25 de maio de 2010

SILVA FILHO, J.P.B Caracterização anatômica de Filgueirasia arenicola (McClure) Guala (POACEAE: BAMBUSOIDEAE: ARTHROSTYLIDIINAE). Dissertação (Mestrado em Botânica). Instituto de Ciências Biológicas, Universidade de Brasília-UNB. Brasília-DF, 2006.

TAPPI - Testing Procedures of Technical Association of the Pulp and Paper Industry. In: TAPPI Standard Method. Atlanta, USA. Cd-Rom, 2001. 